\title{
Exploring the Use of Rapport in Professional Information-Gathering Contexts by Systematically Mapping the Evidence Base
}

\author{
Fiona Gabbert*, Goldsmiths University of London \\ Lorraine Hope, University of Portsmouth \\ Kirk Luther, Carleton University \\ Gordon Wright, Goldsmiths University of London \\ Magdalene Ng, Newcastle University \\ Gavin Oxburgh, Northumbria University
}

This paper is accepted for publication at Applied Cognitive Psychology. This pre-print may not exactly duplicate the final published version.

*Corresponding author. Fiona Gabbert, Department of Psychology, Forensic Psychology Unit, Goldsmiths University of London, New Cross, UK, SE14 6NW. Email:

f.gabbert@gold.ac.uk.

This work was funded by the Centre for Research and Evidence on Security Threats (ESRC Award: ES/N009614/1).

Data availability statement. The datasets referred to in the manuscript are available on the OSF project page: https://osf.io/nsjpz/

Conflict of interest statement. There are no conflicts of interest. 


\begin{abstract}
A growing body of research illustrates consensus between researchers and practitioners that developing rapport facilitates cooperation and disclosure in a range of professional information gathering contexts. In such contexts, rapport behaviors are often intentionally used in an attempt to facilitate a positive interaction with another adult, which may or may not result in genuine mutual rapport. To examine how rapport has been manipulated and measured in professional contexts we systematically mapped the relevant evidence-base in this field. For each of the 35 studies that met our inclusion criteria, behaviors associated with building rapport were coded in relation to whether they were verbal, non-verbal, or paraverbal. Methods to measure rapport were also coded and recorded, as were different types of disclosure. A Searchable Systematic Map was produced to catalogue key study characteristics. Discussion focuses on the underlying intention of the rapport behaviors that featured most frequently across studies.
\end{abstract}

Keywords: rapport; disclosure; information-gathering; investigative interviewing; eyewitness; suspect. 


\section{Exploring the Use of Rapport in Professional Information-Gathering Contexts by Systematically Mapping the Evidence Base}

Within information-gathering contexts such as investigative interviewing, human intelligence debriefings, and the medical field, rapport has been empirically and anecdotally associated with a range of beneficial outcomes - primarily, enhanced information elicitation. While this appears to be a valuable observation, practical implementation of empirical work is impeded by the fact that this assertion is based on vague and divergent definitions of rapport as a construct, the use of a range of experimental manipulations purporting to examine the effects of building rapport, and disparate measurement frameworks. The absence of consensus amongst researchers with respect to how rapport has been defined, manipulated, and measured creates clear challenges for developing effective evidence-based guidelines for the training of rapport development and its measurement. The goal of the current project was to systematically review the extant literature and describe the way in which rapport has been defined and operationalized in the context of information-gathering research to date.

Definitions of rapport are often vague and imprecise, thus leaving room for ambiguity in the ways in which they might be interpreted. For example, rapport has been defined as "The bond or connection between an investigative interviewer and interviewee" (Vallano et al., 2015, p. 369), "A state of communicative alliance" (Abbe \& Brandon, 2013, p. 238), and a "Harmonious, empathetic, or sympathetic relation or connection to another self" (Newberry \& Stubbs, 1990, p. 14). At times, definitions of rapport appear to differ as in practitioner guidelines offered in the UK and US, respectively, "A positive mood between interviewer and interviewee" (Achieving Best Evidence, Home Office, 2011, p. 70) and the establishment of a relationship, "which does not necessarily mean a friendly relationship" (The Army Field 
Manual, Department of the Army, 2006, section 8.4). The absence of a precise definition leaves the term 'rapport' open to considerable interpretation, and very difficult to operationalise or measure with any certainty or consistency, which has significant implications for how it is researched, understood, trained, and practiced.

While there is no widely shared definition of rapport, it is generally accepted that naturally emerging rapport exists at the relationship level, not within an individual (DePaulo \& Bell, 1990; Tickle-Degnen \& Rosenthal, 1990). Tickle-Degnen and Rosenthal's (1990) tripartite theoretical model of rapport remains the most influential in the literature, conceptualizing rapport as having three components: mutual attentiveness, positivity, and coordination. Mutual attentiveness can be described as focused cohesive interaction, involvement, and mutual interest. Positivity includes mutual friendliness, caring, and positive affect. Coordination can be characterized by balance, fluency of interaction, and shared understanding. Each of these three components contain interrelated and distinct features and can develop at different rates. In the early stage of an interaction, Tickle-Degnen and Rosenthal propose that positivity and attentiveness are more heavily weighted than coordination, whereas coordination plays a bigger role in the later stages.

While Tickle-Degnen and Rosenthal's (1990) model provides a useful theoretical framework for understanding the development of naturally emerging rapport in a social context, it is limited in the extent to which it can inform how individuals build rapport in a professional setting. There are some clear differences between social and professional contexts, and it is reasonable to suggest that the process of developing rapport might differ between the two. Social contexts feature the freedom to interact on an equal footing with others who share similar interests and goals, and mutual rapport can develop naturally (or not) over time. In contrast, professional contexts are often characterized by one individual purposefully attempting to develop rapport with another, often within a short period of time. 
Further, rather than being on an equal footing, professional contexts often feature imbalances of power or status, differences in motivation to engage, and differences in desired outcomes from the interaction. For example, in an investigative interview there is an inevitable power imbalance between the interviewer and interviewee. Interviewees may lack motivation to cooperate or may even deliberately resist developing rapport with the interviewer. There are also status imbalances, whereby whether someone is the giver or receiver of information is defined by their formal role in the interaction. As such, the tripartite model is not immediately applicable to building rapport in professional information gathering contexts (though see Abbe \& Brandon, 2013). We believe that drawing the distinction between rapport that occurs naturally and rapport that is engineered is likely to be helpful for practitioners who are expected to attempt to build rapport in professional interactions, and yet might never achieve genuine mutual rapport as traditionally conceptualised.

Given the differences in the development of rapport in professional versus social contexts, we propose the idea that the term 'professional rapport-building' is useful to describe the process of building rapport within a task-oriented professional interaction. Professional rapport-building can be understood as an intentional use of rapport behaviors to facilitate a positive interaction that may, or may not, lead to establishing genuine mutual rapport. Given that all major interviewing and interrogation guidelines acknowledge and endorse the use of rapport-building techniques to facilitate cooperation and communication (e.g., Achieving Best Evidence, Home Office, 2011; Army Field Manual, Department of the Army, 2006; Cognitive Interview, Fisher \& Geiselman, 1992; NICHD Protocol, Lamb et al., 2007; PEACE model, CPTU, 1992), it is a useful endeavor to examine techniques that have been used to build rapport for professional purposes. This requires a focused review of the relevant literature to determine which rapport behavior(s) have been identified as relevant and deemed effective for use in a professional investigative context. 


\section{The Current Review}

In order to examine the use of professional rapport-building in information-gathering contexts, such as an investigative interview or a therapy session where one person is tasked with eliciting information from another, it is necessary to identify empirical research that has manipulated and/or measured selected rapport behaviors and examined their relationship with information disclosure. From the outset of the review, we identified significant inconsistencies and methodological diversity across this body of research and, to address this issue, concluded that an effective method to compare and contrast across studies was to examine the specific rapport behaviors that have featured in each. Taking this approach, it was possible to identify the individual behaviors that researchers had selected to build rapport in a professional context (e.g., which techniques featured in a 'rapport-building script' used by interviewers), and/or which had been selected as rapport behaviors of interest when researchers had coded interactions. For example, Collins et al. (2002) developed a rapportscript for their interviewer to follow, recommending use of a gentle voice tone, relaxed body posture, personalization, and use of the interviewee's name. The rapport script developed by Kieckhaefer et al. (2014) also advised using the interviewee's name, but otherwise recommended that the interviewer shared personal yet unofficial information to encourage reciprocal disclosure, and then to engage in active listening. By identifying the individual rapport behaviors featured in each of these studies, it becomes easier to see the similarities and differences between them. Further, by identifying individual rapport behaviors that have been selected by researchers, it is possible to determine the frequency of each, which may serve as a proxy for perceived value. Last, once individual rapport behaviors have been identified, it is possible to examine the intentions underlying those behaviors, or the functions they serve. For example, the intention behind adopting a gentle voice tone and relaxed body posture is for the interviewer to present an approachable demeanor for the purpose of 
encouraging disclosure. Different behaviors can serve the same function, therefore it is useful (especially for practitioners) to consider which behaviors can be used to build rapport, what they have in common, and why they are effective. In sum, there is value in conducting a systematic and detailed analysis and review of relevant rapport literature to provide an enriched understanding of how rapport has been operationalized in research applied to professional contexts.

Systematic mapping of research on a particular topic allows for transparent and comprehensive evidence collation, visual presentation, and synthesis, which in turn provides an objective picture of the current state of knowledge. Here, we use systematic mapping methodology to examine, compare, and contrast different methods used by researchers to manipulate and measure rapport within an information-gathering context. As an initial stage in our review of the relevant literature, we developed an 'Evidence and Gap Map' (EGM) to facilitate relative areas of concentration and inattention. For example, an EGM enables examination of whether more studies focus on building rapport with suspects rather than witnesses or utilise verbal techniques to build rapport relative to non-verbal techniques. However, while EGMs are able to provide a visual means to examine core features of a relevant body of work, and thus are particularly useful for indicating if sufficient knowledge is available for making policy decisions, they are not a vehicle for synthesizing findings. Thus, we present the EGM and an accompanying summary of findings as supplementary materials for interested readers (https://osf.io/nsjpz/).

Here we focus on providing a detailed overview of the current knowledge base with reference to a 'Searchable Systematic Map' (SSM). The SSM facilitates the cataloguing of study attributes, such as methodologies, independent and dependent variables, and different areas of study focus (see James et al., 2016). As such, this approach effectively helps to map the breadth and depth of relevant literature thus facilitating the identification of knowledge- 
clusters as well as areas within the literature-base that have been neglected. In the current project, we used the systematic map to compare the different study attributes that have been investigated in research examining the use of rapport with adult interviewees within an information-gathering context.

In sum, our objectives were to: (1) identify studies that have manipulated and/or measured rapport within a professional information-gathering context using adult samples; (2) map the existing evidence base; and (3) synthesize the review findings to inform the current state of knowledge regarding the professional use of rapport in information gathering contexts.

\section{Methodology}

The steps for constructing the SSM involved a comprehensive search strategy, screening process, and quality assessment. Following this, data from the studies that met the specified inclusion criteria were coded and presented in the SSM to represent the current knowledge base. Each of these stages are described below.

\section{Search Strategy}

In October 2017, five electronic databases (PsychArticles, PsycInfo, Web of Science, Sociological Abstracts and Proquest Criminology Collection) were searched to identify studies manipulating and/or measuring rapport within an information gathering context. We used the following basic Boolean search string (slightly modified for each database where necessary), where all words must appear in the abstract: $A B$ (rapport* OR empath* OR trust*) AND AB (recall* OR report* N3 info * OR "report*info*" OR report* intell* OR elicit* OR yield* OR disclos* OR retriev*) AND AB (info* OR intell*). The keywords 'empath*' and 'trust*' were initially included as synonyms in the search string to encompass the different ways rapport has been defined, and because researchers in the field frequently use these terms 
when referring to or measuring rapport. In addition to keyword searches, we conducted cited reference searches from key article/s, and circulated requests for relevant published and unpublished studies through professional organizations such as the Society for Applied

Research in Memory and Cognition (SARMAC), and the International Investigative Interviewing Research Group (iIIRG). Grey literature (e.g., unpublished manuscripts and conference proceedings, brief reports, dissertations and theses) was sourced via Google and Twitter searches.

\section{Criteria for Inclusion and Exclusion of Studies}

Laboratory studies were included if they: (a) featured an experimental manipulation of rapport within an information-gathering context (with a comparison condition such as with another rapport technique, control condition, or both); (b) a measurement of rapport, and; (c) a measure that enabled an examination of the effects of rapport on disclosure of information. Field studies and quasi-experimental designs were included if they examined rapport within an information-gathering context and provided sufficient detail regarding methods used to code rapport and any outcome measurements that enabled an examination of the effects of rapport on disclosure of information. Due to our focus on specific interventions and outcomes we did not include qualitative studies, review articles, or books/book sections. Studies focused on building rapport with children were excluded as this is a specific field that addresses unique developmental challenges relating to cognitive and social maturation. Specifically, the body of research on building rapport with children often focuses on the effectiveness of, and use of, age-appropriate techniques (e.g., see the NICHD Protocol, Lamb et al., 2007). Similarly, practitioner guidelines for building rapport often differ according to age (e.g., Achieving Best Evidence, MoJ, 2011). Further, due to the fact that children under 18 years of age are often categorized as 'vulnerable' (due specifically to their age), building 
rapport with them is often conflated with the additional need to provide social support. In the child interviewing literature, rapport and social support have been recognized as distinct constructs (see Saywitz et al., 2015 for an extended discussion).

\section{Search results}

Our initial search yielded over 6,500 hits from the aforementioned databases. In addition, manual searching yielded a further 11 hits, and unpublished/grey articles supplied by authors in the field yielded a further 27. After removing duplicates, 4,758 articles remained for title and abstract screening. The Preferred Reporting Items for Systematic Reviews and Meta-Analyses (PRISMA) flow chart outlines the steps in the screening process, showing the number of articles identified, articles that were included and excluded, as well as reasons for articles being excluded (see Figure 1).

\section{Screening and study selection}

Screening is the process of reviewing identified studies against the inclusion and exclusion criteria for the map to determine eligibility for inclusion. We used a two-stage process: title and abstract review followed by full-text screening. In the first round of screening, titles that had no relevance to rapport and information disclosure or elicitation were eliminated. Abstracts were checked to determine whether: (i) the study manipulated rapport; (ii) the study measured rapport, and; (iii) the outcome variable was information elicitation or disclosure. Those studies that did not meet this inclusion criteria were eliminated at this stage. Furthermore, articles were removed if the abstract revealed that the study did not feature an experimental, quasi-experimental, survey-based, or observational design. In response to peer feedback, we decided to exclude studies examining the role of trust and/or empathy on disclosure, where these studies did not also explicitly focus on rapport, on the grounds that 
those constructs are qualitatively different to rapport and, as such, should not be considered as appropriate synonyms.

Articles that passed the title and abstract screening progressed to full-text screening. Articles were deemed ineligible at this stage if they: (i) did not include an explicit manipulation of rapport; (ii) did not report measures to enable an examination of rapport and/or the effects of rapport; (iii) only measured the intention to disclose rather than disclosure itself, or; (iv) disclosure was limited to only asking people to report their age, sex, and/or name.

Thirty-five articles passed the full-text screening stage and were then coded for: (i) what manipulations of rapport were employed; (ii) what measures of rapport were used, and; (iii) what kind of information elicitation or disclosure the article examined. Screening at each stage (i.e., title and abstract screening, full-text screening) was undertaken by two people independently. A protocol was developed (see 'Instructions for Coders', https://osf.io/nsjpz/), and group training and practice sessions were provided whereby researchers coded the same sample of studies and compared decisions. Calibration (i.e. agreement between researchers) was high prior to commencing the task of categorizing papers according to the inclusion/exclusion criteria. Following training, the lead Research Assistant coded each paper so that there was consistency throughout the process. Trained volunteer researchers coded batches of papers independently. Inconsistencies in coding were infrequent, but when they arose a third researcher independently categorized the paper, and majority decision was accepted.

Figure 1 about here

\section{Developing the Searchable Systematic Map (SSM)}


A SSM summarising core characteristics of the 35 eligible studies was created in Excel in the format of a searchable database. All studies were represented, regardless of whether rapport actually increased, decreased, or had no significant effect on disclosure. The SSM is available as an open-access resource here: https://osf.io/nsjpz/. In the SSM spreadsheet, each of the 35 rows in the database represents an eligible study. The columns represent methodological details relating to each study, including the independent and dependent variables. If a particular rapport behavior featured in four studies or more, then we inferred there was reasonable consensus between researchers that this behavior has potential value for building rapport in a professional context, thus it featured in the SSM as a core verbal or nonverbal rapport behavior. If a rapport behavior featured less frequently then it was positioned in the 'miscellaneous' column. An exception to this rule was applied to studies featuring the rapport-building behaviors of: (i) showing respect; (ii) showing kindness, and; (iii) building trust. Despite four or more studies featuring these behaviors, they were categorized as miscellaneous due to imprecision in the description of how these behaviors were operationalised and/or coded. If a study reported the effects of rapport on whether a suspect confessed, this was categorised as 'amount' of disclosure (as opposed to 'accuracy' of disclosure). However, the SSM also includes a separate column to show which studies reported this particular type of disclosure. See Table 1 for a list of each column category and the related searchable options.

\section{Table 1 about here}

To search the SSM, a user can identify the column/s of interest and select the option/s required from the drop-down menu. For example, if only studies from the Criminal Justice domain are of interest, then this option can be selected from those available in the relevant column, after which the database will only show relevant studies that meet this preference. 


\section{Results of the SSM}

\section{Overview of Study Characteristics}

Of the 35 studies that met the inclusion criteria, $49 \%(n=17)$ were experimental studies (featuring a manipulation of rapport-building), 26\% $(n=9)$ were observational studies, $20 \%$ $(n=7)$ were surveys, and 6\% $(n=2)$ were mixed-method studies. The studies were from a range of fields, including $80 \%(n=28)$ from Criminal Justice featuring investigative interviewers seeking information from suspects, witnesses, or intelligence sources, $11 \%(n=$ 4) from the field of Human-Computer Interaction examining disclosure to virtual interviewers, $6 \%(n=2)$ from Psychology examining interviewer style on disclosure within survey responses, and 3\% $(n=1)$ from the Medical/Health domain that featured a physician interviewing a patient about a medical condition. In terms of the type of interviewee, $60 \%(n$ $=21)$ were suspects (including intelligence sources), $23 \%(n=8)$ were eyewitnesses, $14 \%(n$ $=5)$ were patients/clients, and 3\% $(n=1)$ were survey respondents. The majority of studies $(77 \% ; n=27)$ focused on building rapport and eliciting information in-person, while $23 \%$ ( $n$ $=8$ ) examined building rapport online. Thirty-two studies examined direct interpersonal interaction, whether mediated by computer or phone or in person, while three featured a 'virtual' human (Kang \& Gratch, 2010, 2012, Peiris et al., 2000). All interactions were dyadic.

\section{Rapport behaviors featured}

A range of behaviors was used across the studies to build or observe rapport. These behaviors were coded in relation to whether they were verbal, non-verbal, or para-verbal. Verbal components of rapport included: (i) use of the interviewee's name; (ii) self-disclosure; (iii) showing personal interest; (iv) active listening; and (v) use of empathy. Non-verbal components of rapport included: (i) smiling; (ii) body-posture; (iii) eye-contact; and (iv) head 
nodding. Para-verbal components of rapport included tone of voice. Less frequently used rapport-related manipulations were listed under 'miscellaneous' (e.g., connection building language, mimicry, being kind). The percentage (and number) of studies featuring each rapport behavior are shown in Table 2. It is immediately evident that verbal rapport behaviors have featured more frequently than non-verbal behaviors as a variable that has been manipulated, observed, and/or measured by researchers.

\section{Table 2 about here}

Many of the rapport-building behaviors were operationalised in broadly similar ways across studies. For example, referring to the interviewee by their name and the use of nonverbal rapport behaviors such as smiling, eye-contact, and head-nodding. However, specific instructions about how to implement or code these behaviors were rarely described or otherwise reported. Elsewhere, there were discrepancies in the nature of instructions with respect to particular behaviors. For example, with reference to use of open body language, Collins et al. (2002) referred to relaxed (versus stiff) body posture, while Aruguete and Roberts (2000) operationalized this behavior as leaning forward (versus sitting upright). Regardless of the specific instructions used, however, the intention was to convey an approachable demeanour to create an inviting atmosphere and encourage interaction.

Some consistency was also found in the way verbal rapport-building behaviors were operationalised. For example, regarding use of self-disclosure, Duke et al. (2018a, Study 2) used a rapport script including the following instruction for interviewers, "I'll begin by telling you a few things about myself. .." (p 14), and Vallano and Schreiber Compo (2011) instructed interviewers to disclose personal information to the witness as recommended in the Cognitive Interview (Fisher \& Geiselman, 1992). On the whole, exactly what type of information, how much, and when, interviewers should disclose about themselves was rarely 
described, despite this being an important consideration for training and replication purposes (Ruddle \& Dilks, 2015), and associated negative consequences of getting this approach wrong (Abbe \& Brandon, 2014). An exception was Wachi et al. (2018), who provided direction for their interviewers, asking them to share a memory of a specific experience related to the topic of the interview. Vallano and Schreiber Compo (2011) also provided guidance, instructing their interviewers to match the nature and level of their self-disclosure to that of the interviewee.

Behaviors were categorised as showing a personal interest if the interviewer was able to demonstrate that they were interested in the interviewee as an individual, by encouraging or engaging in topics of discussion that do not necessarily fall within the remit of the interview. For example, Nash et al. (2016) facilitated this rapport technique by training the interviewer in the rapport condition to encourage interviewees to answer questions about themselves, and then respond to their answers by demonstrating an interest; "Oh that's interesting, do you enjoy that? What's your favorite part?" (p. 4) and reciprocating information. Similarly, Duke et al. (2018a, Study 2) instructed interviewers to start the interview by saying "I was wondering if you could please tell me about yourself . .." and "It will help us work together if we know something about each other" (p. 14), after which interviewers were instructed to listen carefully and show that they are interested in the witness and what s/he says. A number of researchers incorporated the strategy of engaging the interviewee in conversation unrelated to the true purpose of the interview to facilitate rapport-building (e.g., Duke et al., 2018b). This 'conversation' creates opportunities for the interviewer and interviewee to share information and get to know one another. This in turn creates opportunities to discover connections and common ground, which serve to promote rapport via highlighting similarities (see Abbe \& Brandon, 2014; Meissner et al., 2015). 
Behaviors used to demonstrate or code for active listening skills often included backchannel responses (e.g. "'uh-huh, yeah') to convey attention and/or interest (e.g., Aruguete \& Roberts, 2000; Vallano \& Schreiber-Compo, 2011). In addition, the use of summaries and paraphrasing has been used to demonstrate attention and understanding (e.g., Moraes, 2013; Walsh \& Bull, 2012). However, it was not uncommon for researchers to refer to active listening without providing a definition or explanation as to the exact behaviors this entailed.

Last, displaying empathy has been recognised as a method to build rapport verbally. Empathy refers to sharing and/or experiencing the feelings of another human being (Davies, 1983). In a professional context such as police interviewing, empathic behaviors include those whereby the interviewer is able to demonstrate that they understand the perspective of the interviewee and/or appreciate the emotions and distress of that person. Displaying empathy is an important component of Alison and colleagues' framework for Observing Rapport-Based Interviewing Techniques (ORBIT). For example, Alison et al. (2013) observed interviews and rated the extent to which the interviewer made an effort to accurately understand the detainee's perspective. Similarly, Walsh and Bull (2012) coded interviews for the extent to which interviewers showed understanding of and concern for suspects' position and emotions.

\section{Measuring rapport}

Measures used for rapport were coded in relation to (i) whether the measure assessed the Interviewer (or the person being disclosed to; $n=24$ ), the Interviewee (or the person disclosing; $n=6)$, or the Interaction itself $(n=23)$, and (iii) whether the study featured a selfreport measure where participants rated their experience of rapport $(n=27)$ and/or an observational measure where trained coders rated pre-defined indicators of rapport-behaviors 
$(n=10)^{1}$. All of the experimental studies that included a direct manipulation of rapport $(n=$ 13) reported higher mean ratings of rapport in the rapport conditions in comparison to 'nonrapport' control groups.

Details about the specific measurements used, such as questionnaires and coding schemes, were also recorded in the SSM. Again, it was apparent that researchers assessed rapport in many different ways, with minimal overlap in the measurement tools used. On only one occasion did a research team use a measure of rapport previously utilized by another team: Oostinga et al. (2018) used Vallano and Schreiber Compo's (2011) measure of rapport. However, Collins and Carthy (2018) were inspired by Tickle-Degnan and Rosenthal's (1990) rapport components model when developing their coding scheme. All other studies developed and used their own measures of rapport. To illustrate how different measurements of rapport are similar or different to one another, example items (where relevant) are reported in the SSM and discussed below.

Where self-report measures of perceived rapport were used, most researchers gave interviewees a questionnaire to complete about the interviewer and/or the interaction. Here there was noticeable overlap in the type of questions asked, which frequently related to detecting the presence of particular rapport behaviors (e.g., the interviewer showed personal interest; showed empathy; smiled), or absence of such behaviors (e.g., the interviewer was nonchalant; impatient; bored). It was less common for self-report questionnaires to ask the interviewee to introspect and report feelings of rapport (e.g., we understood each other well; we respected each other; I felt awkward/uncomfortable/easy telling my story). Perhaps the best-validated measure of rapport in investigative settings is Duke et al.'s (2018a) 'Rapport Scales for Investigative Interviews and Interrogation, Interviewee version (RS3i-I)' which has

\footnotetext{
1 Some studies featured more than one measure and so these measures add to more than 35 .
} 
been shown to have robust internal reliability as well as construct and concurrent validity. This self-report questionnaire measures interviewees' experience of rapport in forensic and intelligence interviews via five scales; Attentiveness, Trust/Respect, Expertise, Cultural Similarity, and Connected Flow. Duke et al. (2018a, Study 2) found that scores on several of the scales were higher when interviewers used rapport-based tactics, and that scores correlated with the amount of information disclosed by interviewees during questioning.

Self-report assessments of rapport are not always possible to obtain however, and there is a need for observational measures to assess the level of rapport within an information gathering interaction. The majority of studies in this category have observed and recorded the presence of specific rapport-building behaviors (e.g., recording instances of showing a personal interest; demonstrating acceptance/empathy; use of name preference, eye-contact, head-nodding, body-posture). Thus, there is an overlap in the behaviors that are used to manipulate rapport, self-evaluate rapport, and observe rapport. Perhaps the most comprehensive approach to observing and assessing rapport at present is Alison et al.'s (2013; 2014) taxonomy for Observing Rapport Based Interviewing Techniques (ORBIT). This measurement tool differs from many others in that it does not attempt to identify instances of discrete rapport behaviors. Instead, it comprises more holistic evaluations of rapport-building skills and interpersonal style of communication that are assessed throughout the interview. These different approaches to measuring rapport either by identifying specific rapportbehaviors or evaluating communication and connection across specified periods of time highlight the range of attempts that have been made to capture and reliably quantify rapport. To date, however, the extent to which any observational measures of rapport are valid and reliable indicators that an interviewee has experienced rapport has proved difficult to quantify and no research in the SSM examined the relationship between measures of observed and self-reported rapport (for discussion, see Vallano \& Compo, 2015). This is somewhat 
surprising given the generally accepted consensus that genuine rapport is mutual.

Summarising across the findings from observational studies, $90 \%$ report a positive relationship between the use of rapport behaviors and enhanced disclosure. However, in the absence of self-report measurement, it is not possible to confirm the extent to which genuine rapport is necessary to achieve desirable outcomes in professional contexts.

In sum, there is limited consistency across studies in the way rapport is measured. Researchers have largely sought to quantify the presence of verbal and non-verbal rapportrelated behaviors used by interviewers. Some measures have attempted to capture the experience of rapport, but there is sizeable variation in the types of questions asked to achieve this. However, the observation that: (i) when used as a manipulation check, participants in experimental rapport conditions endorse more rapport-related characteristics than control condition counterparts, and; (ii) higher scores on measures of rapport (observed and selfreport tools) are associated with greater cooperation and yield from interviewees, lends credence to the notion that these measures meaningfully capture the essence of rapport. Without further research, including direct comparisons between different measures of rapport, it is difficult to conclude which are the most effective.

\section{Outcome variables relating to disclosure}

Finally, we coded for the type of disclosure reported in terms of quantity and/or quality (accuracy) measures. Assessing how rapport influences the amount of information reported is vital, as an increase in disclosure provides the interviewer with more information, evidence, and potential lines of enquiry, as well as providing more details to challenge, where necessary. Knowing the accuracy of the information reported is also a valuable measure to ensure that any increases in disclosure do not simply reflect a lower threshold of reporting accompanied by a lower accuracy rate overall. However, relatively fewer studies reported 
accuracy data. This is likely due to the number of field studies included where ground truth (and, therefore, accuracy) is generally unknown.

The amount of information reported was measured in a variety of ways across studies, ranging from a tally of the total number of words reported (Kieckhaefer et al., 2014; Vallano \& Schreiber Compo, 2011), to a more selective process of coding for specific types of information such as the number of points of evidence or items of intelligence reported (Alison et al., 2013), or the amount of investigation-relevant information obtained during the interview (Collins et al., 2002). Duke et al.'s (2018a) Shared Information Rating Scale (SIRS) comprised a check-list of facts about the video that could have been mentioned by an interviewee. Under the category of 'amount reported' we also included studies using preexisting measures of depth of disclosure such as Jourard and Lasakow's (1958) SelfDisclosure Scale. If studies reported confession data $(n=15)$, it was coded as amount (rather than accuracy) of disclosure. The SSM includes a column to facilitate the identification of these particular studies that have examined the effects of rapport on confession evidence.

\section{Narrative overview of findings}

Of the 35 studies in the review, 32 reported full or partial support for positive effects of rapport on disclosure. For example, Collins et al. (2002) and Nash et al. (2016) examined the effect of rapport on eyewitness recall and found that participants in the rapport condition reported more items of accurate information than participants in comparison conditions. In an analysis of real-world suspect interviews, Walsh and Bull (2012) found that interviewers skilled in establishing and maintaining rapport in line with PEACE interview guidelines were five times more likely to obtain positive interview outcomes, such as eliciting a comprehensive account and/or a full and frank confession (for similar results see Wachi et al., 2018). Partial support for positive effects of rapport on disclosure was reported by Vallano 
and Schreiber Compo (2011) who found that rapport-building increased recall accuracy and decreased susceptibility to post-event misinformation, but only in response to open-ended questions, and not in response to cued-recall questions.

Three studies did not find a clear or expected relationship between the use of rapportbehaviors and disclosure. For example, Buene et al. (2010) examined whether the use of different information gathering behaviors by police officers affects the provision of information by suspects from high and low-context cultures and found that, contrary to expectations, high context suspects responded to 'active listening' by refusing to provide information (in comparison to low-context suspects). Duke et al. (2018) examined whether a 'cooperation-focused' interview approach facilitated the development of rapport more than a 'withholding-focused' interview approach that was expected to elicit anxiety. Surprisingly, participants reported high rapport with the interrogator in both conditions, more information was reported by participants in the withholding-focused interview and rapport did not mediate the relationship between the interview condition and the number of details reported. Finally, Kelly et al. (2015) used real-world data to examine reported and actual rates of different interrogation methods, of which one was rapport and relationship building, and their association with suspect confession or denials. This analysis suggested that while rapport was used often, it had no significant impact on interrogation outcomes.

While this narrative overview provides a snapshot of findings reported by studies included in the review, there are two important caveats to note. First, it was not always possible to determine whether these are direct or indirect effects. For example, researchers rarely examine rapport ratings as a mediator/moderator to the amount of information reported (although see Brimbal et al., 2019). Second, establishing the overall effect size for rapport requires a meta-analysis, which is beyond the scope of this review. 


\section{Synthesizing findings: integrating themes identified in the literature}

Identifying and comparing the verbal, non-verbal, and para-verbal rapport behaviors used most frequently by researchers to facilitate the task of eliciting information sheds considerable light on the literature to date. However, in addition to examining individual rapport behaviors, it is perhaps more prudent to consider the underlying intention, or function that each behavior serves. Each of the rapport behaviors identified have been used to achieve, or are associated with, a desired outcome. Different behaviors can have the same outcome, therefore, it is useful (especially for practitioners) to consider not only which behaviors can be used to build rapport but why they are effective. Based on the literature, we propose that each of the rapport-behaviors used across studies can be meaningfully grouped according to one of three core functions, each of which has been used to support the development of rapport: (i) personalizing the interview; (ii) presenting an approachable demeanor, and; (iii) paying attention (see Table 3). Note that Table 3 depicts only the rapport behaviors that have featured most frequently across the studies included in the review; undoubtedly, there are other behaviors that also serve these goals, and that some behaviors also serve a secondary function, for example, lowering an interviewee's anxiety or stress levels.

\section{Table 3 about here}

Personalizing the interview is a strategy used to build rapport that has featured across a number of studies. The prevalence is not surprising as personalizing the interview is recommended in the Cognitive Interview (Fisher \& Geiselman, 1992). This strategy is believed to help the interviewer build a personal connection with the interviewee by expressing interest in them as an individual. We propose that of the rapport behaviors featured across studies, using someone's preferred name, showing an interest in them as a person, sharing information about oneself, and highlighting some similarity with the interviewee 
where appropriate, are all techniques that have been used to promote personal engagement. Taking steps to get to know the interviewee as an individual has a number of benefits, including enhanced understanding of the interviewee's motivations, that can benefit perspective-taking and interview strategy (Abbe \& Brandon, 2014; Alison et al., 2013; Galinsky et al., 2008). Further, it enables the interviewer to find, and highlight, similarities or common ground with the interviewee, which has been found to predict both cooperation and disclosure (Goodman-Delahunty \& Howes, 2016). In a survey administered to US law enforcement officers, Vallano et al. (2015) found the most common rapport-building technique reported was to engage in small talk to find common ground. Sharing information about oneself (interviewer self-disclosure) is an additional technique that can be used to show similarities and to encourage reciprocal disclosure from the interviewee (Collins \& Miller, 1994; Duke et al., 2018a; Vallano \& Schreiber Compo, 2011). In a review of therapeutic practice, Henretty and Levitt (2010) found that $90 \%$ of therapists use self-disclosure as a tool to promote client disclosure, show similarities, and develop a therapeutic alliance.

Researchers who have used the method of personalizing an interview to build rapport have often used more than one of the behaviors to demonstrate an interest in the interviewee. For example, in Kieckhaefer et al.'s study (2014) interviewers used a rapport script that encouraged them to use the interviewee's name, ask personal questions (e.g., "Tell me about your family"), and then to display interest in the information disclosed in response. Wachi et al. (2018) used similar techniques, and observed that this approach was more effective in eliciting (true) confessions than presenting suspects with actual evidence. A growing body of research is examining other methods and measures of personalization such as coordination of interviewer/interviewee responses (e.g., Beune et al., 2010) and language style matching (e.g., Taylor \& Thomas, 2008). 
Moving to the second grouping of behaviors operationalized to gain rapport, we noted that smiling, use of open body language, and using an appropriate (conversational or neutral as opposed to hostile) tone of voice, are all behaviors that have featured across studies as methods of presenting an approachable demeanor. Again, studies have often used more than one of these behaviors to facilitate rapport. For example, in Collins et al.'s (2002) study, interviewers in the rapport condition followed a script encouraging them to speak with a gentle tone, adopt a relaxed body posture, and be generally friendly. Presenting oneself as approachable serves to invite interaction by creating a genial and positive atmosphere. Extensive research shows how sensitive we are to verbal and non-verbal communication in interactions that can influence a range of responses such as how much we like the other person, how willing we are to engage with them, and reciprocal demeanor - whereby the way someone is perceived promotes a similar response (Uleman et al., 2008; Zebrowitz, 2017).

In the third grouping of rapport-gaining behaviors, we noted that paying attention during an interaction was often used to develop and maintain professional rapport. This grouping included active listening, use of empathy, eye-contact, and head-nodding as behaviors that serve to signal interest, understanding, and appreciation of the information being shared, which in turn encourages continued disclosure. Perhaps unsurprisingly, TickleDegnen and Rosenthal (1990) also highlighted the importance of 'mutual attentiveness' as a core component in their model of rapport for social interactions. However, in professional contexts whereby behaviors are used to build rapport, the mutuality of attentiveness is not necessary, albeit desired. Rather, we propose that it is important to demonstrate attentiveness regardless of whether the other person reciprocates. Eye-contact and head-nodding are two powerful non-verbal behaviors to demonstrate attention and understanding (e.g., Davidhizar, 1992). However, 'active' behaviors involve not merely absorbing information but remembering and responding to it appropriately over the course of an interaction. Therefore, 
active listening and use of empathy are more cognitively demanding as these require the receiver to demonstrate that they are processing the information received. Additionally, empathy requires perspective-taking skills to demonstrate that the receiver is able not just to understand what is being said, but how the person might be feeling. Regardless of how attention is demonstrated, if these behaviors signal attention and engagement, then an interviewee will likely be encouraged to continue the interaction; if not, interactions can break down and the amount of information disclosed by the interviewee is reduced (Abbe \& Brandon, 2013; Holmberg \& Madsen, 2014; Walsh \& Bull, 2012).

In sum, we argue that the rapport-behaviors identified across studies in the SSM are manifestations of one of three categories of behavioral practices commonly used to establish a rapport in a professional context; personalizing an interview, presenting an approachable demeanor, and paying attention to what is being said. Twenty-six studies within the SSM used rapport behaviors to support at least two of these practices. Contemplating the underlying intention of a behavior may be helpful when training people how to build rapport for the purpose of information elicitation, as with this information in hand, interviewers can select which behaviors are likely to be most effective for achieving a particular goal or outcome. This choice of which behaviors to use might depend on the interview format; for example, open body language cannot be conveyed in a phone interview, but tone of voice can be used to present an approachable demeanor instead. The choice might also depend on an interviewer's particular style, or where cultural norms suggest that a particular behavior might not have the desired effect (e.g., use of direct eye-contact can signal engagement and interest in Western cultures, but may be considered rude or aggressive in some other cultures; AlKrenawi \& Graham, 2000).

\section{General Discussion}


Rapport helps create bonds and form relationships with others. As such, it is an adaptive social skill. However, select behaviors to build rapport are also trained and used in professional settings for the purposeful goal of building relationships in order to facilitate cooperation. While a larger body of research has examined the benefits of rapport in naturalistic social interactions, the studies contained in our SSM enable us to examine how researchers have utilised rapport behaviors specifically to facilitate the task of eliciting information in a professional context.

\section{Rapport building behaviors in professional contexts}

Our systematic approach to identifying discrete verbal and non-verbal behaviors used by researchers to manipulate and measure rapport enabled us to systematically describe, compare, and contrast the existing literature. However, this approach involves reducing rapport, a complex social phenomenon, to isolated atomistic or component parts and, consequently, raises a number of important discussion points. First, while we have identified behaviors that have been used in professional contexts to attempt to build rapport, we do not endorse the claim that these behaviors are necessary or sufficient for the development of genuine mutual rapport, which is likely more than the sum of these component parts. Professional rapport-building is simply an intentional use of rapport behaviors in an attempt to facilitate a positive interaction with another person that might or might not lead to establishing genuine rapport. We propose that distinguishing between professional rapportbuilding and naturally emerging rapport has important implications for both theoretical and applied advancements in knowledge and understanding of rapport. We encourage more observational research of experienced and skilled practitioners in ecologically valid settings to learn more about both. 
Second, while the outcome of this systematic review supports the use of certain verbal and non-verbal behavioral correlates of rapport, practitioners should be aware that ineffective use carries risks. An obvious danger of cultivating rapport via deliberately using selected behaviors, rather than allowing rapport to develop and manifest naturally, is that the recipient detects the deliberate nature of the rapport-gaining behaviors, and the relationship becomes damaged (Abbe \& Brandon, 2014). For example, displaying signs of attentiveness without really listening, or over-using rapport behaviors, may quickly appear disingenuous or insincere. While research has addressed which rapport behaviors might be effective in an information elicitation context, it has rarely addressed how best to operationalize these behaviors (though see Abbe \& Brandon, 2014) or sustain rapport across extended or repeated interactions, despite practitioners voicing a need for such practical guidance (e.g., Russano et al., 2014). We urge researchers to examine the consequences of maladaptive use of rapport behaviors, as this is a clear gap in the literature. Similarly, insights into how best to recover from a loss of rapport has rarely been addressed in the literature. A recent exception is work by Oostinga at al. (2018) examining the effectiveness of different response strategies on an interviewer's ability to recover trust and rapport after a communication breakdown. Further research in this area is clearly critical to understand the dynamic nature of rapport in a professional setting.

\section{Limitations}

Systematically mapping the evidence base has facilitated a thorough analysis of research that has examined rapport in an information elicitation context. However, our review is not without limitations. First, a major concern when conducting a review of a body of literature is that relevant papers might have been missed or overlooked. We implemented a range of measures to avoid this. However, it is inevitable that a small number of potentially relevant studies use terms that do not match our search strategy and, as a result, were not 
included. Second, to ensure transparency and consistency across the review process we deliberately only coded the rapport behaviors named and defined as such by the authors. We did not assume or infer the presence of rapport behaviors where rapport was not explicitly stated by the authors as having been manipulated or observed. Further, where rapport training or rapport scripts were used, it is necessarily a matter of trust that interviewers implemented the behaviors outlined. This means that our Systematic Map may not reflect all of the rapport behaviors that actually featured within studies. We call on researchers to provide full and detailed information in Method sections to facilitate reliable conclusions being drawn in future systematic reviews. Third, we acknowledge that the method we used to enable us to compare and contrast across studies presents a rather reductionist view of rapport and limits the extent to which we can examine the dynamic nature of rapport as it develops over time, across topic, and between different people. While this facet of rapport is discussed in the wider literature (e.g., Tickle-Degnen \& Rosenthal, 1990), very few empirical studies have addressed it directly. Three exceptions are Walsh and Bull's (2012) study that examined rapport maintenance throughout entire interviews with suspects, Oostinga at al.'s (2018) study that examined rapport recovery after a communication breakdown, and Collins and Carthy's (2018) study that examined rapport during the beginning, middle, and end of an interview, finding that rapport was positively correlated with the amount of disclosure in the middle and end of the interview. This topic is certainly a gap in our understanding that requires considered research attention.

\section{Implications for future research}

One outcome of this systematic review process is that it clearly illuminates a number of future directions for researchers to consider, in addition to the more methodological issues discussed above. First, the majority of the studies featured in the maps are 'Western-centric' drawing on theories developed with respect to interpersonal interactions and communication 
in Western contexts or using data drawn from White, Educated, Industrialized, Rich, and Democratic (WEIRD) samples (Henrich et al., 2010); we therefore call for more research examining the extent to which the rapport behaviors discussed here generalise to building rapport in other cultures. It is likely that some rapport behaviors will not be universally effective due to cultural factors. For example, Schug et al. (2010) found that the efficacy of self-disclosure in building relationships differed for cultures featuring high relational mobility (such as the UK and US) versus low (e.g., Japan). Second, broadly speaking, most studies that have focused on building rapport with suspects have used an observational approach while studies focusing on witnesses are often experimental, where the presence/absence of rapport has been manipulated. We recommend that researchers consider a balanced use of both methods in witness and suspect interview contexts in order to provide a comprehensive understanding of the benefits of rapport. Third, seven studies referred to 'showing respect' when describing how rapport was manipulated or measured, but without any detail as to which behaviors might be used for this purpose. Given the intuitive importance of promoting a respectful relationship as a step towards building rapport, we call on researchers to be as precise and detailed as possible in their description of how respect can be operationalised and/or coded. Last, we recommend that researchers investigate the separate and combined effects of different rapport behaviors, to build a knowledge base of which behaviors can be used interchangeably dependent upon context (e.g., online vs. face-to-face), which are more effective in certain situations (e.g., building a relationship vs. being attentive), and which behaviors are most effective at different stages of an interaction (e.g., as per Tickle-Degnen \& Rosenthal's model, 1990).

\section{Conclusion}

Using the SSM methodology it has been possible to identify the rapport behaviors selected for manipulation and/or measurement by researchers in an information elicitation 
context. Rapport is an inherently complex and dynamic social process, and it is worth considering that the phenomenon of rapport might be immune to meaningful or complete description at any individual unit of verbal, non-verbal, or paraverbal behavior. However, when considering the underlying intention of each of the behaviors identified when mapping the literature, it has been possible to group rapport behaviors in relation to the goal served, namely; (i) personalizing an interview, (ii) presenting an approachable demeanor, and (iii) paying attention. We urge future researchers to refine this work by examining not only how best to cultivate rapport for professional purposes, but also how to effectively train the development, maintenance, and recovery of rapport in professional contexts. 


\section{References}

Studies included in the systematic review are preceded by an asterisk.

Abbe, A., \& Brandon, S. E. (2013). The role of rapport in investigative interviewing: A review. Journal of Investigative Psychology and Offender Profiling, 10(3), 237-249. https://doi.org/10.1002/jip.1386

Abbe A., \& Brandon, S. E. (2014). Building and maintaining rapport in investigative interviews, Police Practice and Research: An International Journal, 15(3), 207-220. https://doi.org/10.1080/15614263.2013.827835

* Alison, L. J., Alison, E., Noone, G., Elntib, S., \& Christiansen, P. (2013). Why tough tactics fail and rapport get results: Observing rapport-based interpersonal techniques (ORBIT) to generate useful information from terrorists. Psychology, Public Police, and Law, 19(4), 411-431. https://doi.org/10.1037/a0034564

Alison, L., Alison, E., Noone, G., Elntib, S., Waring, S., \& Christiansen, P. (2014). The efficacy of rapport-based techniques for minimizing counterinterrogation tactics amongst a field sample of terrorists. Psychology, Public Policy, and Law, 20(4), 421430. https://doi.org/10.1037/law0000021

Al-Krenawi, A., \& Graham, J. R. (2000). Culturally sensitive social work practice with Arab clients in mental health settings. Health \& Social Work, 25, 9-22. https://doi.org/10.1093/hsw/25.1.9

* Aruguete, M. S., \& Roberts, C. A. (2000). Gender, affiliation, and control in physicianpatient encounters, Sex Roles, 42(1-2), 107-118.

https://doi.org/10.1023/a:1007036412480 
* Beune, K., Giebels, E. \& Sanders, K. (2009). Are you talking to me? Influencing behaviour and culture in police interviews. Psychology, Crime \& Law, 15, 597617.doi:10.1080/10683160802442835

* Beune, K., Giebels, E., \& Taylor, P. J. (2010). Patterns of interaction in police interviews: The role of cultural dependency. Criminal Justice and Behavior, 37(8), 904-925. https://doi.org/10.1177/0093854810369623

Brandon, S. E., Wells, S. \& Seale, C. (2018). Science-based interviewing: Information elicitation. Journal of Investigative Psychology and Offender Profiling, 15(2), 133-148. https://doi.org/10.1002/jip.1496

Brimbal, L., Dianiska, R. E., Swanner, J. K., \& Meissner, C. A. (2019). Enhancing cooperation and disclosure by manipulating affiliation and developing rapport in investigative interviews. Psychology, Public Policy, and Law, 25(2), 107-115. https://doi.org/10.1037/law0000193

Carkhuff, R. K. (1969). Helping and Human Relations. New York, NY: Holt, Rinehart and Winston.

Central Planning \& Training Unit (1992). A guide to interviewing. Harrogate: CPTU.

* Collins, K., \& Carthy, N. (2018). No rapport, no comment: The relationship between rapport and communication during investigative interviews with suspects. Journal of Investigative Psychology and Offender Profiling, 16(1), 1-14. https://doi.org/10.1002/jip.1517

* Collins, R., Lincoln, R., \& Frank, M. G. (2002). The effect of rapport in forensic interviewing. Psychiatry, Psychology, and Law, 9(1), 69-78. https://doi.org/10.1375/pplt.2002.9.1.69 
Collins, N. L., \& Miller, L. C. (1994). Self-Disclosure and Liking: A Meta-Analytic Review. Psychological Bulletin, 116(3), 457-475. https://doi.org/10.1037/0033-2909.116.3.457

Colquitt, J. A., LePine, J. A., Piccolo, R. F., Zapata, C. P., \& Rich, B. L. (2012). Explaining the justice-performance relationship: Trust as exchange deepener or trust as uncertainty reducer? Journal of Applied Psychology, 97(1), 1-15. https://doi.org/10.1037/a0025208

Davidhizar, R. (1992). Interpersonal communication: a review of eye contact. Infection Control \& Hospital Epidemiology, 13, 222-225.

Davies, M. H. (1983). Measuring individual differences in empathy: Evidence for a multidimensional approach. Journal of Personality and Social Psychology, 44(1), 113126. https://doi.org/10.1037/0022-3514.44.1.113

Department of the Army. (2006). Army Field Manual FM2-22.3: Human collector operations. Washington, DC: Author.

DePaulo, B. M., \& Bell, K. L. (1990). Rapport is not so soft anymore. Psychological Inquiry, 1(4), 305-308. https://doi.org/10.1207/s15327965pli0104_6

* Duke, M. C., Wood, J. M., Bollin, B., Scullin, M., \& LaBianca, J. (2018a). Development of the rapport scales for investigative interviews and interrogations (RS3i), interviewee version. Psychology, Public Policy, and Law, 24(1), 64-79. https://doi.org/10.1037/law0000147

* Duke, M. C., Wood, J. M., Magee, J., \& Escobar, H. (2018b). Effectiveness of Army Field Manual interrogation approaches for educing information and building rapport. Law and Human Behavior, 42(5), 442-457. https://doi.org/10.1037/lhb0000299

Fisher, R. P., \& Geiselman, R. E. (1992). Memory-enhancing techniques for investigative interviewing: The cognitive interview. Springfield, IL: Charles C. Thomas. 
Galinsky, A. D., Maddux, W. W., Gilin, D., \& White, J. B. (2008). Why it pays to get inside the head of your opponent: The differential effects of perspective taking and empathy in strategic interactions. Psychological Science, 19(4), 378-384.

https://doi.org/10.1111/j.1467-9280.2008.02096.x

Goodman-Delahunty, J., \& Howes, L. M. (2016). Social persuasion to develop rapport in high-stakes interviews: qualitative analyses of Asian-Pacific practices. Policing and Society, 26(3), 270-290. https://doi.org/10.1080/10439463.2014.942848

* Goodman-Delahunty, J., \& Martschuk, N. (2018). Securing reliable information in investigative interviews: Coercive and noncoercive strategies preceding turning points. Police Practice and Research, 1-20. doi:10.1080/15614263.2018.1531752

* Goodman-Delahunty, J., Martschuk, N., \& Dhami, M. K. (2014). Interviewing high value detainees: Securing cooperation and disclosures. Applied Cognitive Psychology, 28, 883-897. doi:10.1002/acp.3087

Henrich, J., Heine, S., \& Norenzayan, A. (2010). The weirdest people in the world? Behavioral and Brain Sciences, 33, 61-83. doi:10.1017/S0140525X0999152X

Henretty, J. R., \& Levitt, H. M. (2010). The role of therapist self-disclosure in psychotherapy: A qualitative review. Clinical Psychology Review, 30(1), 63-77. https://doi.org/10.1016/j.cpr.2009.09.004

* Henson, R., Cannell, C. F., \& Lawson, S. (1976). Effects of interviewer style on quality of reporting in a survey interview. The Journal of Psychology, 93, 221-227. doi:10.1080/00223980.1976.9915816

* Holmberg, U., \& Christianson, S-Å. (2002). Murderers' and sexual offenders’ experiences of police interviews and their inclination to admit or deny crimes. Behavioral Sciences and the Law, 20, 31-45. doi:10.1002/bsl.470 
* Holmberg, U., \& Madsen, K. (2014). Rapport operationalized as a humanitarian interview in investigative interview settings. Psychiatry, Psychology and Law, 21(4), 591-610. https://doi.org/10.1080/13218719.2013.873975

Home Office. (2011). Achieving Best Evidence in Criminal Proceedings: Guidance on Interviewing Victims and Witnesses, and Using Special Measures. London: Author.

James, K. L., Randall, N. P., \& Haddaway, N. R. (2016). A methodology for systematic mapping in environmental sciences. Environmental Evidence, 5(7), 1-13. https://doi.org/10.1186/s13750-016-0059-6

* Johnson, W. T., \& Delamater, J. D. (1976). Response effects in sex surveys. The Public Opinion Quarterly, 40, 165-181. doi:10.1086/268285

Jourard, S. M., \& Lasakow, P. (1958). Some factors in self-disclosure. Journal of Abnormal and Social Psychology, 56(1), 91-98. https://doi.org/10.1037/h0043357

* Kang, S-H., \& Gratch, J. (2012). Socially anxious people reveal more personal information with virtual counselors that talk about themselves using intimate human back stories. Studies in Health and Technology Informatics, 181, 202-206.

* Kang, S., H., \& Gratch, J. (2010). Virtual humans elicit socially anxious interactions' verbal self-disclosure. Computer Animations and Virtual Worlds, 21, 473-482. doi:10.1002/cav.345

* Kebbell, M., Alison, L., Hurren, E. \& Mazerolle, P. (2010). How do sex offenders think the police should interview to elicit confessions from sex offenders? Psychology, Crime \& Law, 16, 567-584. doi:10.1080/10683160902971055 
* Kelly, C. E., Redlich, A. D., \& Miller, J. C. (2015). Examining the meso-level domains of the interrogation taxonomy. Psychology, Public Policy, and Law, 21, 179-191. doi:10.1037/law0000034

* Kieckhaefer, J. M., Vallano, J. P., \& Schreiber Compo, N. (2014). Examining the positive effects of rapport building: When and why does rapport building benefit adult eyewitness memory? Memory, 22(8), 1010-1023. https://doi.org/10.1080/09658211.2013.864313

Lamb, M. E., Orbach, Y., Hershkowitz, I., Esplin, P. W., \& Horowitz, D. (2007). A structured forensic interview protocol improves the quality and informativeness of investigative interviews with children: A review of research using the NICHD Investigative Interview Protocol. Child Abuse \& Neglect, 31(11-12), 1201-1231. https://doi.org/10.1016/j.chiabu.2007.03.021

* Leahy-Harland, S., \& Bull, R. (2017). Police strategies and suspect responses in real-life serious crime interviews. Journal of Police and Criminal Psychology, 32, 138-151. doi:10.4324/9781315169910-15

Meissner, C. A., Kelly, C. E., \& Woestehoff, S. A. (2015). Improving the Effectiveness of Suspect Interrogations. Annual Review of Law and Social Science, 11, 211-233. https://doi.org/10.1146/annurev-lawsocsci-120814-121657

Memon, A., Meissner, C. A., Fraser, J. (2010). The Cognitive Interview: A meta-analytic review and study space analysis of the past 25 years. Psychology, Public Policy, and Law, 16(4), 340-372. https://doi.org/10.1037/a0020518

MoJ (2011). Achieving best evidence in criminal proceedings: Guidance on interviewing victims and witnesses, and using special measures. London: Her Majesty's Stationary Office. 
* Moraes, S. L. (2013). The positive relationship between police rapport tactics and investigative interviewing outcomes. Unpublished thesis. Fielding Graduate University.

* Nash, R. A., Nash, A., Morris, A., \& Smith, S. L. (2016). Does rapport-building boost the eyewitness eyeclosure effect in closed questioning? Legal and Criminological Psychology, 21(2), 305-318. https://doi.org/10.1111/lcrp.12073

Newberry, J. J., \& Stubbs, C. A. (1990). Advanced interviewing techniques. Glynco, Georgia: Bureau of Alcohol Tobacco and Firearms National Academy.

* Oostinga, M. S. D., Giebels, E., \& Taylor, P. J. (2018). Communication error management in law enforcement interactions: a receiver's perspective. Psychology, Crime \& Law, 24(2), 134-155. https://doi.org/10.1080/1068316X.2017.1390112

* Peiris, D. R., Gregor, P., \& Alm, N. (2000). The effects of simulating human conversational style in a computer-based interview. Interacting with Computers, 12, 635-650. doi:10.1016/S0953-5438(00)00028-X

* Redlich, A. D., Kelly, C. E., \& Miller, J. C. (2014). The who, what, and why of human intelligence gathering: Self-reported measures of interrogation methods. Applied Cognitive Psychology, 28, 817-828. doi:10.1002/acp.3040

Richardson, B. H., Taylor, P. J., Snook, B., Conchie, S. M., \& Bennell, C. (2014). Language style matching and police interrogation outcomes. Law and Human Behavior, 38(4), 357-366. https://doi.org/10.1037/lhb0000077

Roter, D. L., \& Larson, S. (2002). The Roter interaction analysis system (RIAS): Utility and flexibility for analysis of medical interactions. Patient Education and Counseling, 46(4), 243-251. https://doi.org/10.1016/S0738-3991(02)00012-5 
Rotter, J. (1967). A New Scale for the Measurement of Interpersonal Trust. Journal of Personality, 35, 651-665. https://doi.org/10.1111/j.1467-6494.1967.tb01454.z

Ruddle, A., \& Dilks, S. (2015). Opening up to disclosure. The Psychologist, 28(6), 458-461.

Russano, M. B., Narchet, F. M., Kleinman, S. M., \& Meissner, C. A. (2014). Structured interviews of experienced HUMINT interrogators. Applied Cognitive Psychology, 28(6), 847-859. https://doi.org/10.1002/acp.3069

Saywitz, K., Larson, R., Hobbs, S., Wells, C., 2015. Developing rapport with children in forensic interviews: systematic review of experimental research. Behavioural Sciences and the Law 33, 372-389. https://doi.org/10.1002/bs1.2186

Schug, J., Yuki, M., \& Maddux, W. (2010). Relational Mobility Explains Between- and Within-Culture Differences in Self-Disclosure to Close Friends. Psychological Science, 21, 1471-1478. https://doi.org/10.1177/0956797610382786

* Snook, B., Brooks, D., \& Bull, R. (2015). A lesson on interrogations from detainees: predicting self-reported confessions and cooperation. Criminal Justice and Behavior, 42, 1243-1260. doi:10.1177/0093854815604179

* Sun. H. (2014). Rapport and its impact of disclosure of sensitive information in standardized interviews. Unpublished thesis.

Thom, D. H., Ribisl, K. M., Stewart, A. L., \& Luke, D. A. (1999). Further validation and reliability testing of the Trust in Physician Scale. The Stanford Trust Study Physicians. Medical Care, 37(5), 510-517. https://doi.org/10.1097/00005650-199905000-00010

Tickle-Degnen, L., \& Rosenthal, R. (1990). The nature of rapport and its nonverbal correlates. Psychological Inquiry, 1, 285-293. https://doi.org/10.1207/s15327965pli0104_1 
Uleman, J. S., Saribay, S. A., \& Gonzalez, C. M. (2008). Spontaneous inferences, implicit impressions, and implicit theories. Annual Review of Psychology, 59, 329-360. https://doi.org/10.1146/annurev.psych.59.103006.093707

Vallano, J. P., \& Compo, N. S. (2015). Rapport-building with cooperative witnesses and criminal suspects: A theoretical and empirical review. Psychology, Public Policy, and Law, 21(1), 85-89. https://doi.org/10.1037/law0000035

* Vallano, J. P., \& Schreiber Compo, N. (2011). A comfortable witness is a good witness: Rapport building and susceptibility to misinformation in an investigative mock-crime interview. Applied Cognitive Psychology, 25(6), 960-970. https://doi.org/10.1002/acp.1789

Vallano, J. P., Evans, J. R., Schreiber Compo, N., \& Kieckhaefer, J. M. (2015). RapportBuilding During Witness and Suspect Interviews: A Survey of Law Enforcement. Applied Cognitive Psychology, 29(3), 369-380. https://doi.org/10.1002/acp.3115

* Wachi, T., Kuraishi, H., Watanabe, K., Otsuka, Y., Yokota, K., \& Lamb, M. E. (2018). Effects of rapport building on confessions in an experimental paradigm. Psychology, Public Policy, and Law, 24(1), 36-47. https://doi.org/10.1037/law0000152

* Wachi, T., Watanabe, K., Yokota, K., Otsuka, Y., Kuraishi, H., \& Lamb, M. (2014). Police interviewing styles and confessions in Japan. Psychology, Crime \& Law, 20, 673-694. doi:10.1080/1068316X.2013.854791

* Wachi, T., Watanabe, K., Yokota, K., Otsuka, Y., \& Lamb, M. E. (2016). Japanese interrogation techniques from prisoners' perspectives. Criminal Justice and Behavior, 43, 617 - 634. doi:10.1177/0093854815608667 
* Walsh, D., \& Bull, R. (2012). How do interviewers attempt to overcome suspects' denials? Psychiatry, Psychology and Law, 19(2), 151-168. https://doi.org/10.1080/13218719.2010.543756

* Walsh, D. \& Bull, R. (2012). Examining rapport in investigative interviews with suspects: Does its building and maintenance work? Journal of Police and Criminal Psychology, 27, 73-84. doi:10.1007/s11896-011-9087-x

Zebrowitz, L. A. (2017). First impressions from faces. Current Directions in Psychological Science, 26(3), 237-242. https://doi.org/10.1177/0963721416683996 
Table 1

Column categories featured in the Systematic Map and related searchable options.

\begin{tabular}{|c|c|}
\hline Column categories & Searchable options \\
\hline \multicolumn{2}{|c|}{ Study information and methods } \\
\hline Full APA reference & Various \\
\hline Year of publication & Various \\
\hline Field & $\begin{array}{l}\text { Criminal justice; Human-computer interaction; } \\
\text { Medical/Health/ Psychology }\end{array}$ \\
\hline Participants & Children; Adults \\
\hline Paradigm & In-person; Online \\
\hline Type of study & Experimental; Mixed methods; Observational; Survey \\
\hline Experimental manipulation & Various \\
\hline Interviewee Type & $\begin{array}{l}\text { Eyewitnesses, Suspects, Patients/Clients, Survey } \\
\text { Respondents }\end{array}$ \\
\hline
\end{tabular}

Verbal rapport components featured

\begin{tabular}{|ll|}
\hline Use of interviewee's name & YES (vs. blank) \\
\hline Self-disclosure & YES (vs. blank) \\
\hline Show personal interest & YES (vs. blank) \\
\hline Active listening & YES (vs. blank) \\
\hline Use of empathy & YES (vs. blank) \\
\hline
\end{tabular}

Non- verbal rapport components featured

\begin{tabular}{|ll|}
\hline \multicolumn{1}{|l}{ Smiling } & YES (vs. blank) \\
\hline Body posture & YES (vs. blank) \\
\hline Eye contact & YES (vs. blank) \\
\hline Head nodding & YES (vs. blank) \\
\hline Para-verbal (Tone) & YES (vs. blank) \\
\hline Miscellaneous & Various \\
\hline Measures of rapport & \\
\hline Target of assessment & Interviewers rating self; Interviewees rating self; \\
& Interviewees rating Interviewer; Interviewees rating \\
\hline Type of test & interaction; Researchers coding interaction. \\
\hline Measures + example items & Self-report; Observation \\
\hline Outcome variables & \\
\hline Yield / Amount & YES (vs. blank) \\
\hline Yield / Accuracy & YES (vs. blank) \\
\hline
\end{tabular}


Table 2

Percentages and frequencies of studies featuring rapport behaviors. Note, percentages add to more than 100\% because studies frequently featured more than one rapport behavior.

\begin{tabular}{|lll|}
\hline Rapport behaviors used & \% of studies & (No. of studies) \\
\hline Verbal & & $(23)$ \\
\hline Active listening & & $(18)$ \\
Show personal interest / reciprocity & $56 \%$ & $(14)$ \\
Use of self-disclosure & $40 \%$ & $(13)$ \\
Empathic responses & $37 \%$ & $(7)$ \\
Use of interviewee's name & $20 \%$ & $(5)$ \\
\hline Non-verbal & & $(4)$ \\
\hline Smiling & $14 \%$ & $(4)$ \\
Open body language & $11 \%$ & $(4)$ \\
Eye-contact & $11 \%$ & \\
\hline Head-nodding & $11 \%$ & $(8)$ \\
\hline Para-verbal & $23 \%$ & \\
\hline Tone of voice & & \\
\hline
\end{tabular}


Table 3

Percentages and frequencies of studies featuring behaviors that function to support three key methods to build rapport. Note, percentages add to more than $100 \%$ because studies frequently featured more than one rapport behavior.

\begin{tabular}{lll}
\hline Function of rapport behavior used $\quad \%$ of studies $\quad$ (No. of studies)
\end{tabular}

\section{Personalizing/relationship building}

Show personal interest / reciprocity

$51 \%$

Use of self-disclosure

$40 \%$

Use of interviewee's name

$20 \%$

\section{Presenting an approachable demeanor}

Tone of voice $23 \%$

Smiling

$14 \%$

Open body language

$11 \%$

\section{Paying attention}

Active listening

$66 \%$

Empathic responses

$37 \%$

Eye-contact

$11 \%$

Head-nodding

$11 \%$ 


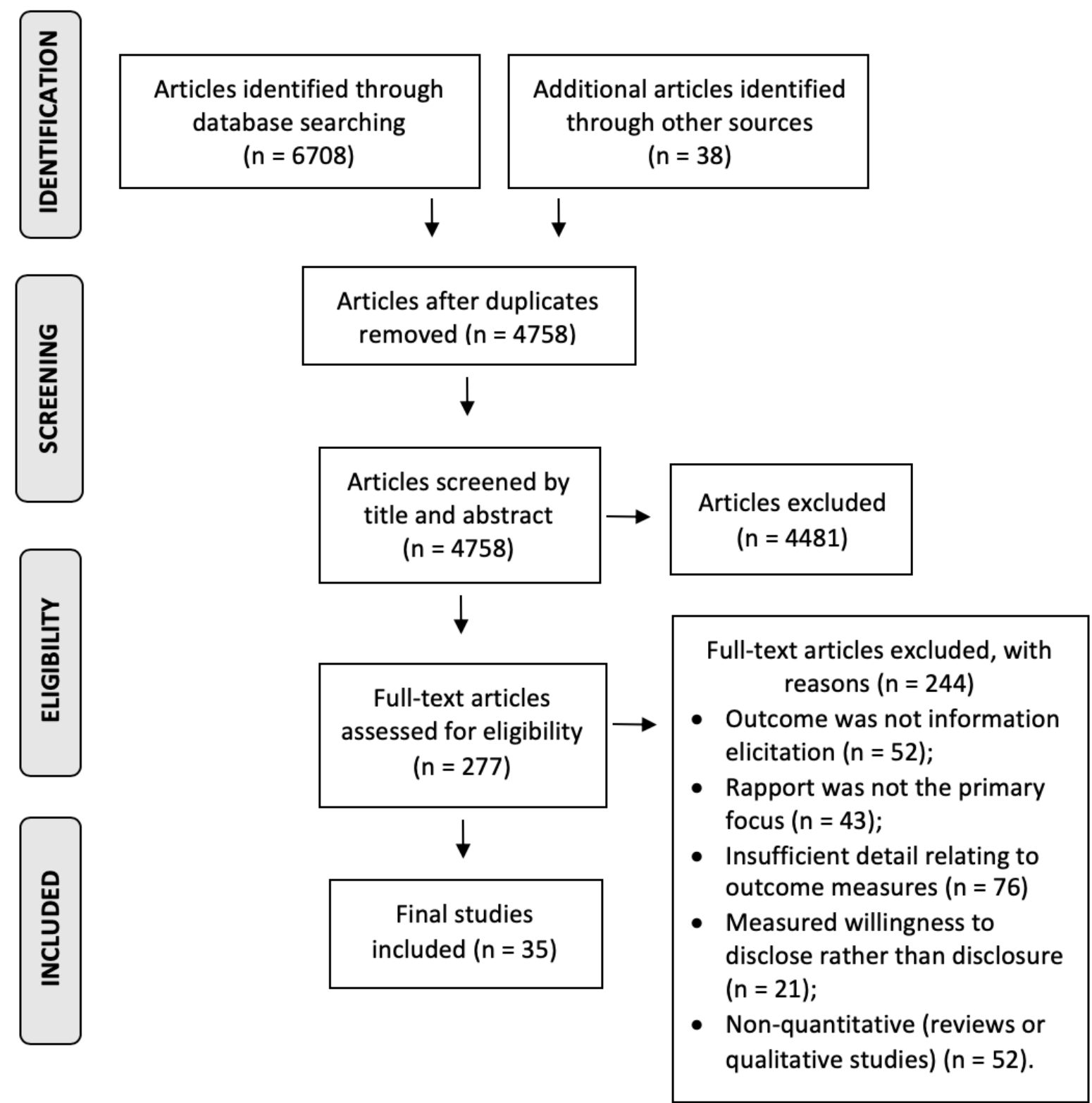

Figure 1. PRISMA flow chart outlining key phases. Note, thirty-three articles in total; two articles had more than one study included. 